\title{
WEAK GRAVITATIONAL LENSING AND CLUSTER MASS ESTIMATES
}

\author{
Christopher A. Metzler, ${ }^{1}$ Martin White, ${ }^{1,2}$ Michael Norman, ${ }^{2,3}$ and Chris Loken ${ }^{4}$ \\ Received 1999 April 16; accepted 1999 May 20; published 1999 June 22
}

\begin{abstract}
Hierarchical theories of structure formation predict that clusters of galaxies should be embedded in a weblike structure, with filaments emanating from them to large distances. The amount of mass contained within such filaments near a cluster can be comparable to the collapsed mass of the cluster itself. Diffuse infalling material also contains a large amount of mass. Both of these components can contribute to the cluster weak lensing signal. This "projection bias" is maximized if a filament lies close to the line of sight to a cluster. Using large-scale numerical simulations of structure formation in a $\Lambda$-dominated cold dark matter model, we show that the projected mass typically exceeds the actual mass by several tens of percent. This effect is significant for attempts to estimate cluster masses through weak lensing observations and will affect weak lensing surveys aimed at constructing the cluster mass function.
\end{abstract}

Subject headings: cosmology: theory — galaxies: clusters: general — gravitational lensing

\section{INTRODUCTION}

Clusters of galaxies are excellent cosmological probes. Their size suggests that their constituents provide a fair sample of the universe. Their structure and hydrodynamic state provide information on their formation and evolution and thus on models of structure formation. Measurements of the abundance of clusters of a given mass constrain the amplitude of mass fluctuations in the universe; measurements of abundance evolution can be used to constrain the mass density $\Omega_{m}$.

Many of these approaches depend upon some knowledge of the mass, or mass distribution, of the cluster. Most techniques for measuring cluster masses are based upon some equilibrium assumption which relates the cluster mass to an observable such as the temperature of the intracluster plasma or the velocity dispersion of cluster galaxies. Recently, however, it has become feasible to measure the surface density distribution of a cluster through observations of weak gravitational lensing of the background galaxy field by the cluster. An attractive quality of this technique is that no assumptions about the dynamical or thermodynamical state of the cluster components need be made. In other words, weak lensing analyses probe the mass distribution directly.

However, analyses of the mass distribution of a cluster drawn from weak lensing observations are not without problems (for a recent review, see Mellier 1999). Many of these relate to details of the procedure adopted to go from the observed ellipticity distribution to the mass or from instrumental effects. We will not discuss these in this Letter. We are interested here in the degree to which weak lensing mass estimates of clusters are affected by lensing from material outside but nearby the cluster. This is a source of systematic error that is not well understood (although it has been alluded to in earlier work, e.g., Miralda-Escude 1991; Cen 1997; Wambsganss, Cen, \& Ostriker 1998; Reblinsky \& Bartelmann 1999).

\footnotetext{
${ }^{1}$ Harvard College Observatory, 60 Garden Street, Cambridge, MA 02138; cmetzler@cfa.harvard.edu,mwhite@cfa.harvard.edu.

${ }^{2}$ Departments of Physics and Astronomy, University of Illinois, 1002 West Green Street, Champaign, IL 61801.

${ }^{3}$ Laboratory for Computational Astrophysics, National Center for Supercomputing Applications, University of Illinois, 405 North Mathews Avenue, Urbana, IL 61801; norman@ncsa.uiuc.edu.

${ }^{4}$ Department of Physics and Astronomy, University of Missouri, Columbia, MO 65211; cloken@hades.physics.missouri.edu.
}

Since clusters form in overdense regions, the volume surrounding a cluster is likely to contain infalling overdense matter (Gunn \& Gott 1972). This infalling matter could add to an observed lensing signal and result in an overestimate of the cluster mass. It is possible that this bias could be quite severe. In modern hierarchical models of structure formation, such as the cold dark matter (CDM) model, numerical simulations imply that clusters form primarily at the intersections of filaments in a web of cosmic structure, accreting additional diffuse mass and smaller collapsed objects that drain along these filaments. It is thus reasonable to expect a beaded filamentary structure to surround most clusters of galaxies. Tentative observational evidence of filamentary structure near clusters has been reported recently (Kull \& Boehringer 1999; Kaiser et al. 1999). A filament lying near the line of sight will also lens the background galaxies and therefore contribute spuriously to the lensing signal. If the observed lensing signal were attributed solely to the cluster, the inferred cluster mass could be much larger than its actual mass.

In this Letter, we use mock clusters from numerical simulations to explore the significance of the systematic mass overestimation induced by the additional lensing signal of both diffuse and filamentary material near the cluster. We find that this effect can be quite significant and must be considered when evaluating the results of lensing mass reconstruction techniques. In the next section, we describe the numerical data and our procedure for evaluating the errors introduced into cluster mass estimates by nearby material. Section 3 describes the results of our analyses. We discuss these results and outline plans for future study at the end.

\section{METHOD}

Weak lensing mass reconstruction techniques produce a map of shear or convergence. These are integrals of the mass along the line of sight times a projection kernel. This kernel is quite wide in the redshift direction, scaling as $D_{L} D_{L S} / D_{S}$, where $D_{L}$ is the distance from the observer to the lens, $D_{S}$ from the observer to the source, and $D_{L S}$ from the lens to the source (Mellier 1999). ${ }^{5}$ Under the assumption that the cluster is the most massive object along the line of sight and is well localized

\footnotetext{
${ }^{5}$ For a distribution of source distances, one takes an appropriate integral of this expression.
} 
TABLE 1

Parameters for the Three Simulated Clusters

\begin{tabular}{lccc}
\hline \hline & \multicolumn{3}{c}{ NUMBER } \\
\cline { 2 - 5 } PARAMETER & 0 & 2 & 4 \\
\hline$R_{\text {sphere }}\left(h^{-1} \mathrm{Mpc}\right) \ldots \ldots \ldots$ & 12.9 & 15.1 & 14.9 \\
$r_{200}\left(h^{-1} \mathrm{Mpc}\right) \ldots \ldots \ldots \ldots$ & 3.14 & 2.76 & 2.60 \\
$M_{200}\left(h^{-1} 10^{15} M_{\odot}\right) \ldots \ldots$. & 2.16 & 1.47 & 1.23 \\
$M_{>70}\left(h^{-1} 10^{15} M_{\odot}\right) \ldots \ldots$. & 2.41 & 3.11 & 2.35 \\
$M_{>10}\left(h^{-1} 10^{15} M_{\odot}\right) \ldots \ldots$. & 3.24 & 4.24 & 3.37 \\
$M_{\text {tot }}\left(h^{-1} 10^{15} M_{\odot}\right) \ldots \ldots \ldots$ & 3.89 & 5.09 & 4.15 \\
\hline
\end{tabular}

Note. $-R_{\text {sphere }}$ is the size of the sphere, centered on the cluster, which we consider in this work, $r_{200}$ is the three-dimensional radius defined in eq. (1) and $M_{200}$ the mass enclosed. $M_{>70}$ and $M_{>10}$ are masses cutting out particles above thresholds of 70 and 10 times local density respectively (see text). $M_{\text {tot }}$ is the total mass in the sphere.

in space (the thin-lens approximation), the convergence map is proportional to the projected surface density map of the lensing cluster itself.

Any additional mass located near the cluster and along the line of sight will also contribute to the lensing signal. Since the kernel is such a slowly varying function of distance, material even at large distances from the cluster will contribute within the thin lens approximation. For a source at $z=1$ and a cluster at $z \sim 0.5$, the kernel changes by only $1 \%$ within $\pm 40 h^{-1} \mathrm{Mpc}$ of the cluster in a universe with $\Omega_{m}=0.3=$ $1-\Omega_{\Lambda}$, with similar results in other cosmologies. As a result, weak lensing observations will probe the projected density of a cluster plus all of the material in its vicinity. Note that this "nearby" material is essentially "at" the redshift of the cluster for the purposes of lensing and so cannot be distinguished by using extra information such as source redshifts.

To study the effect of the surrounding mass upon the projected mass inferred from lensing observations of the simulated clusters, we have examined the mass distribution around several clusters of galaxies extracted from a large cosmological simulation. The simulated clusters were taken from the X-Ray Cluster Data Archive of the Laboratory for Computational Astrophysics of the National Center for Supercomputing Applications and the Missouri Astrophysics Research Group of the University of Missouri. To produce these clusters, a particlemesh $N$-body simulation incorporating adaptive mesh refinement was performed in a volume $256 h^{-1} \mathrm{Mpc}$ on a side. Regions in which clusters formed were identified; for each cluster, the simulation was then rerun (including a baryonic fluid) with finer resolution grids centered upon the cluster of interest. In the adaptive mesh refinement technique, the mesh resolution dynamically improves as needed in high-density regions. The "final" mesh scale at the highest resolution was $15.6 h^{-1} \mathrm{kpc}$, allowing good resolution of the filamentary structure around the cluster. Inside the cluster, the characteristic separation between the smallest mass particles, given by $d=$ $\left(4 \pi r_{200}^{3} / 3 N\right)^{1 / 3}$ with $N$ the number of particles inside the region, was approximately $86 \mathrm{kpc}$ for all three clusters examined here. The code is described in detail in Norman \& Bryan (1999).

The clusters used here were extracted at $z=0$ from simulations of a $\Lambda \mathrm{CDM}$ model, with parameters $\Omega_{m}=0.3, \Omega_{B}=$ 0.026, $\Omega_{\Lambda}=0.7, h=0.7$, and $\sigma_{8}=0.928$. In this Letter, we observe these clusters as if they were at $z=0.5$. In future work, we plan to investigate the dependence of these results on cosmology and on cluster redshift.

Since the number density of rich clusters is approximately $\phi_{*} \sim 10^{-5} \mathrm{Mpc}^{-3}$, the typical separation between them is $\phi_{*}^{-1 / 3} \sim 40 h^{-1} \mathrm{Mpc}$. This is a characteristic scale for filaments:

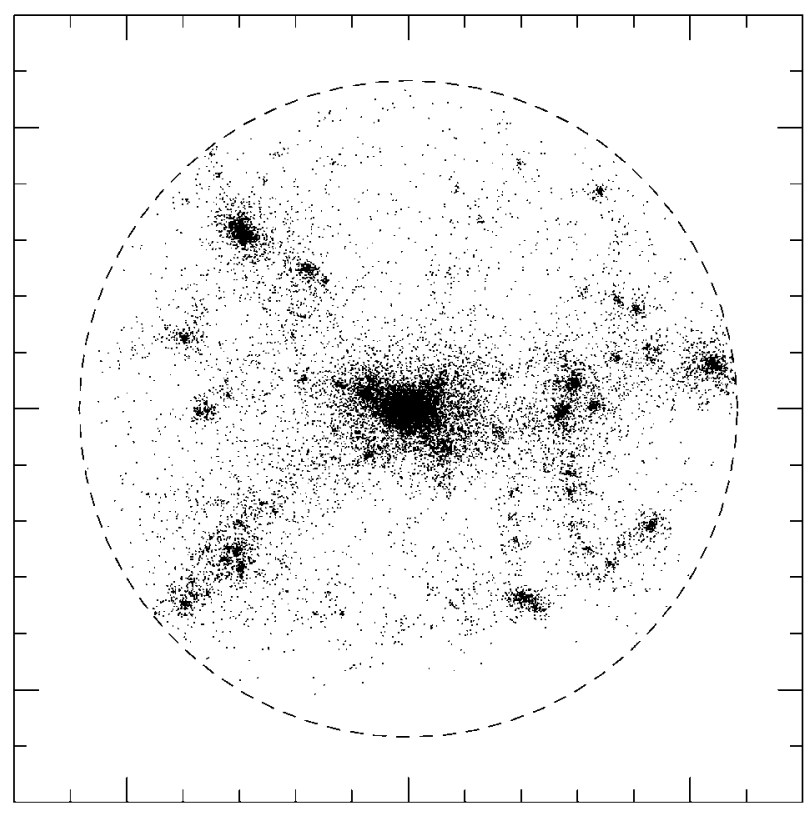

FIG. 1.-Cluster 4, with a fraction of the particles projected onto the $x-z$ plane. Note the filamentary structure, with clumps beading up in the filaments. The dashed circle marks the sphere of radius $R_{\text {sphere }}$ to within which we have restricted our analysis (see text).

volumes containing a cluster and with one-dimensional extent $\sim 40 h^{-1}$ Mpc should also contain much of the nearby filamentary structure. Three such volumes, containing a rich cluster (clusters 0, 2, and 4) as well as satellites and filaments, were extracted from the archive. We selected clusters that did not appear to be mergers or have a large secondary mass concentration nearby. Such systems might be excluded observationally from studying, for instance, the galaxy line-of-sight velocity distribution. For each volume, the "center" of our cluster was determined using a maximum-density algorithm. Since the extracted volumes were not spherical, it was possible that some lines of sight could contain more mass than others simply by geometry. To avoid such biases, we restrict our analysis to particles that lay within the largest sphere, centered on the cluster, which was contained entirely within the extracted volume. The radii of these spheres $R_{\text {sphere }}$ are listed in Table 1, along with other properties of the clusters. Note that these radii are large compared to the projected values of $r_{200}$ obtained for each cluster; thus, no significant radial surface density gradient is introduced by a decreasing chord length through the sphere with radius. It is also important to note that since our volumes are by necessity limited, our results should be interpreted as a lower limit to the size of the effect; the magnitude of the lensing kernel is still significant at the edge of our spherical volume.

Each of the clusters we examined was surrounded by a large amount of mass. Most of this material appeared by eye to be collapsed into "beads" along a filamentary structure, although a small number of clumps could be found outside the filaments. We show a projection of a fraction of the points from the simulation of cluster 4 in Figure 1. The filamentary structure and satellites are easily evident. Note that this filamentary structure extends well beyond our radius $R_{\text {sphere }}$. No single projection can show the full three-dimensional nature of the structure, in which the filamentarity is even more apparent. Since much of this mass is at low density, it is unlikely it would be a site for galaxy formation or otherwise emit light. Thus, this structure 
would not be easy to constrain by observations of redshifts near the cluster.

We observed each of the three selected clusters from 10,000 randomly chosen viewing angles. For each cluster and viewing angle, the projected surface density map was constructed and used to estimate $r_{200}$, the radius within which the mean interior density contrast is 200 . In three dimensions, this radius is defined in terms of the enclosed mass by

$$
M\left(<r_{200}\right)=200\left(\frac{4 \pi}{3}\right) \Omega_{m} \rho_{\text {crit }} r_{200}^{3} .
$$

The projected estimate of $r_{200}$ was extracted from the surface density map by considering the radius of the circle, centered on the cluster, which contained the amount of mass given by equation (1) above, i.e.,

$$
\int_{0}^{2 \pi} d \theta \int_{0}^{r_{200}} R d R \Sigma(R, \theta)=M\left(<r_{200}\right)
$$

with $\Sigma(R, \theta)$ the surface density on the map in terms of a twodimensional radius $R$. This radius was compared to the cluster's true $r_{200}$, extracted from the three-dimensional mass distribution. The ratio of the projected mass to true mass is given simply by the cube of the ratio of the estimated value of $r_{200}$ to the true value. For each cluster, a value of this ratio was obtained for each viewing angle.

\section{RESULTS}

Our main result is displayed in Figure 2, where we show the distribution of projected versus "true" cluster mass in each of the three simulated clusters for our 10,000 randomly chosen viewing angles. We have checked that the features in the histogram do not come from shot noise due to discrete particles in the simulation. However, the "spikiness" is due to a discrete number of objects in the neighborhood of the cluster. A small lump of matter near the cluster will project entirely within $r_{200}$ for a fraction of the lines of sight. For any such line of sight, the effect on the projected value of $r_{200}$ is identical.

We expect the ratio $M_{200} / M_{\text {true }}$ to be greater than unity, since only additional mass can be included in the projection. The size of the smallest offset from unity for clusters 0 and 4 is approximately twice what would be expected for material uniformly distributed at the mean density. This suggests that matter near the cluster is itself clustered and at higher than mean density. The width of the histogram in Figure 2, as a fraction of the true mass, depends on the true mass of the cluster. Although we have only a few clusters in this study, it appears that the mass in nearby material is not proportional to the cluster mass; thus, the relative effect of this material is smaller the larger the cluster. The total mass in the sphere, $M_{\mathrm{tot}}$, is also listed in Table 1 for reference.

The signal shown in Figure 2 comes from (primarily filamentary) material outside the cluster and is not the well-known projection effect arising from cluster asphericity. To verify this, we repeated the procedure described above for a subset of particles aimed at selecting the cluster alone. This was done by first selecting out particles with a local density contrast of greater than 70 (chosen because density profiles near $r^{-2}$ reach a local density contrast near 70 at a mean interior density contrast of 200); a small sphere containing the cluster but little nearby material was then cut out of this subset. The histogram

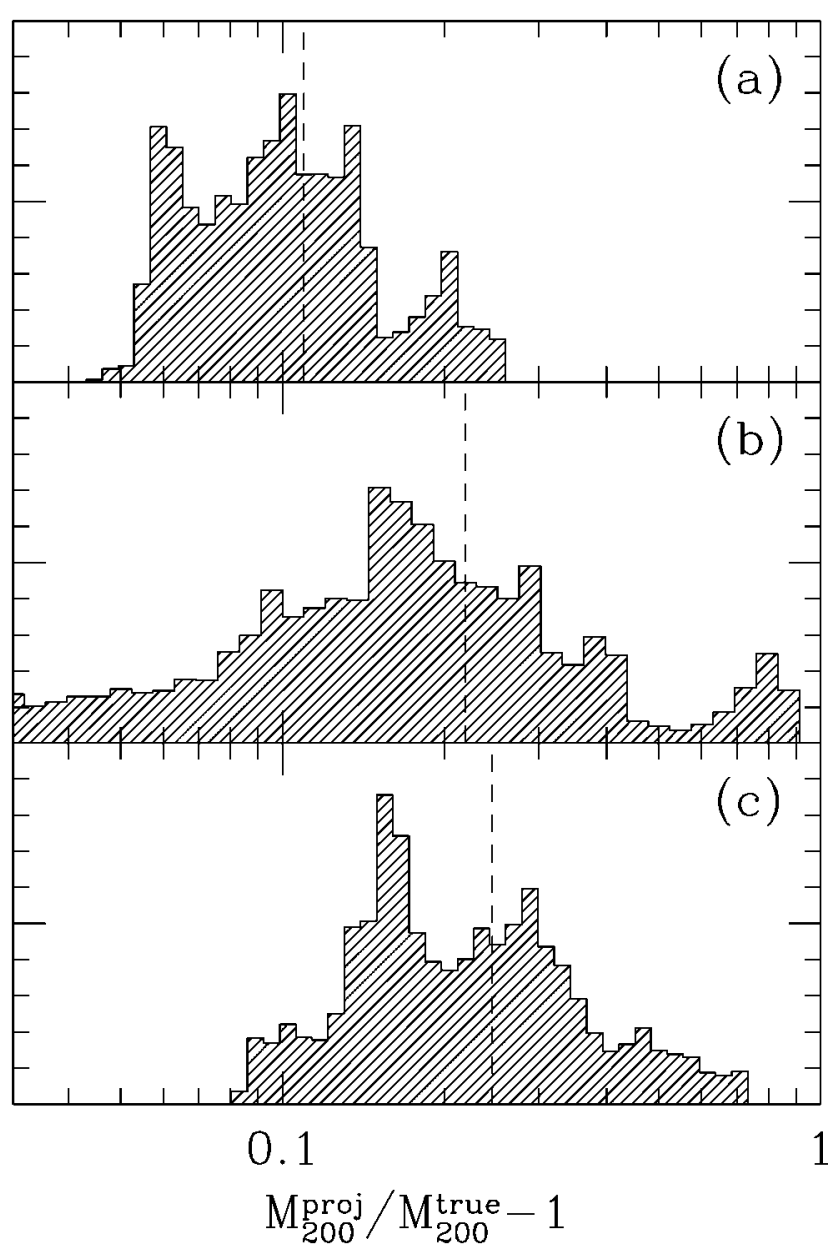

FIG. 2.-Ratio of projected to actual mass within $r_{200}$ for our three simulated clusters, for 10,000 random viewing angles each. In all cases nearby mass (primarily in filaments) has biased the projected mass distribution to higher values. The typical bias is a few tens of percent (panels $b$ and $c$ ), with less of an effect on massive clusters (panel $a$ ). The vertical dashed line marks the mean of the distribution.

produced by viewing the clearly prolate cluster at a large number of randomly chosen viewing angles produced a much narrower distribution, with a maximum offset of less than $10 \%$ in the mass ratio and a mean offset of approximately half that value.

While it is beyond the scope of this Letter to perform a detailed modeling of any observational weak lensing strategy, we show in Figure 3 two sample profiles obtained from aperture densitometry on our noiseless projected mass maps. Specifically, for cluster 4, we show the profile along the lines of sight giving the largest and smallest $r_{200}$ for comparison. The $\zeta$ statistic is the mean value of the convergence $k$ within a disk of radius $r_{1}$ minus the mean value within an annulus $r_{1} \leq r \leq r_{2}$ (Fahlman et al. 1994; Kaiser 1995). Here we calculate $\zeta$ directly from the projected mass, although observationally it would be computed from the tangential shear. We have taken $r_{2}=$ $800^{\prime \prime}$, within the half-degree field of view typical of new large CCD cameras. We have explicitly checked that reducing the radius to half this value does not change our result.

In calculating the convergence $\kappa$, the cluster was again assumed to be at a redshift of 0.5 , with the lensed sources at a redshift of 1.0. In any real observation, of course, the lensed sources will span a range of redshifts. For material very close 


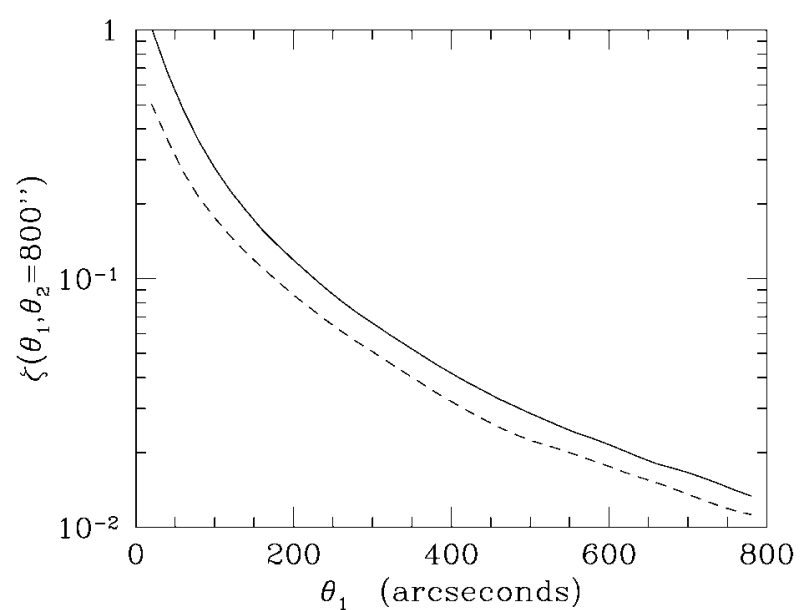

FIG. 3.-Profile $\zeta\left(r_{1}, r_{2}\right)$ (see text) for cluster 4 along two lines of sight with extremal values of $r_{200}$. We have used $r_{2}=800^{\prime \prime}$ in making this figure; reducing this radius by a factor of 2 does not change the result. Note that both profiles appear smooth and well behaved, even though they differ in mass by a factor of 1.7 .

to the cluster, such as here, this will not affect our conclusions, and the error introduced by incorrectly modeling the redshift distribution of the background sources is not the subject of this work. In addition to being easy to estimate, the $\zeta$ statistic is robust and minimizes contamination by foreground mass (Mellier 1999) because it is insensitive to the sheet mass degeneracy. This does not, however, remove the sensitivity to clustered material, as can be seen in Figure 3. The mass which would be inferred from Figure 3 along two lines of sight differ by a factor of 1.7 .

While the distribution of the projection effect varies from cluster to cluster, it seems clear that positive biases in projected mass of $20 \%$ are typical, with biases above $30 \%$ not uncommon. Cen (1997) performed a similar study and found smaller positive bias, typically of only $5 \%-10 \%$. However, Cen subtracted off projected mass at the background since such material does not contribute to the lensing signal; as noted above, this mass accounts for half the offset in our smallest observed biases. More significantly, the masses compared by Cen were measured within an aperture of fixed size. A positive discrepancy in the ratio $M_{\text {proj }} / M_{200}$ measured within the three- dimensional $r_{200}$ implies that the projected estimate of $r_{200}$ is larger, and the resulting estimate of $M_{200}$ larger still.

Furthermore, we emphasize again that these estimates are in fact lower limits; some lines of sight through the untruncated volume produced overestimates as large as $80 \%$ or more. While appropriate modeling of a mean density profile outside $r_{200}$ (drawn perhaps from simulations such as these) can be used to produce an unbiased estimator, the width of these histograms implies a large amount of scatter around such an estimator of the true (unprojected) mass. It is clear that this effect can be quite significant and must be taken into account when attempting to understand the results of mass reconstruction analyses.

\section{CONCLUSIONS}

Clusters of galaxies are part of the large-scale structure of the universe, and observations of them should be considered within this context. The filamentary structure near a cluster can contain a reasonable fraction of the mass of a cluster in tenuous material. Should a filament lie close to the line of sight to a cluster, it will contribute to the weak lensing signal and positively bias the projected mass. We have shown that such a bias could easily be $30 \%$ (see Fig. 2).

Weak lensing remains one of the best methods for determining the mass of clusters of galaxies. However, methods that obtain the mass from estimates of the projected surface density must consider the effect outlined in this Letter. This is clearly of particular significance for attempts to determine the mass function of clusters directly through surveys of weak lensing-determined masses.

We have not attempted to address the detailed question of how much this filamentary signal would affect a particular reconstruction algorithm; the answer is no doubt algorithm dependent. We hope to return to this issue in future work as well as to consider the effect of cosmological model and evolution with cluster redshift.

The authors would like to thank Greg Bryan and Greg Daues for assistance in understanding the archive data and Gary Bernstein, Gordon Squires, and Albert Stebbins for useful conversations on lensing. We would also like to thank the anonymous referee for bringing relevant prior work to our attention. This research was supported by the NSF.

\section{REFERENCES}

Cen, R. 1997, ApJ, 485, 39

Fahlman, G., Kaiser, N., Squires, G., \& Woods, D. 1994, ApJ, 437, 56

Gunn, J. E., \& Gott, J. R. 1972, ApJ, 176, 1

Kaiser, N. 1995, ApJ, 439, L1

Kaiser, N., Wilson, G., Luppino, G., Kofman, L., Gioia, I., Metzger, M., \& Dahle, H. 1999, ApJ, submitted (astro-ph/9809268)

Kull, A., \& Boehringer, H. 1999, A\&A, 341, 23
Mellier, Y. 1999, ARA\&A, in press

Miralda-Escude, J. 1991, ApJ, 380, 1

Norman, M. L., \& Bryan, G. L. 1999, in Numerical Astrophysics '98, ed. S. Miyama \& K. Shibata (Dordrecht: Kluwer), in press

Reblinsky, K., \& Bartelmann, M. 1999, A\&A, in press

Wambsganss, J., Cen, R., \& Ostriker, J. P. 1998, ApJ, 494, 29 